https://helda.helsinki.fi

\title{
Mifepristone may inhibit the midcycle gonadotropin surge at both ovarian and pituitary sites of action : [correspondence]
}

\section{Heikinheimo, Oskari}

2005

Heikinheimo, O , Leminen , R \& Raivio , T 2005 , ' Mifepristone may inhibit the midcycle gonadotropin surge at both ovarian and pituitary sites of action : [correspondence] ', Fertility and Sterility : official journal of the American Fertility Society , vol. 84 , no. 5 , pp. 1545-1546 . https://doi.org/10.1016/j.fertnstert.2005.04.058

http://hdl.handle.net/10138/297727

https://doi.org/10.1016/j.fertnstert.2005.04.058

publishedVersion

Downloaded from Helda, University of Helsinki institutional repository.

This is an electronic reprint of the original article.

This reprint may differ from the original in pagination and typographic detail.

Please cite the original version. 


\title{
Mifepristone may inhibit the midcycle gonadotropin surge at both ovarian and pituitary sites of action
}

\begin{abstract}
The ability of mifepristone to delay ovulation is thought to be based on the antigonadotropic effects of the drug. In the current work, late-follicular phase administration of mifepristone resulted in decrease in both circulating FSH and inhibin B, suggesting both central and ovarian sites of action. (Fertil Steril ${ }^{\circledR} 2005 ; 84$ : 1545-6. (C2005 by American Society for Reproductive Medicine.)
\end{abstract}

Preovulatory administration of mifepristone postpones ovulation by inhibiting the midcycle surge of gonadotropin secretion $(1,2)$, a property that has been employed in emergency contraception (3). Recently, a novel protocol for controlled ovarian hyperstimulation $(\mathrm{COH})$ using a combination of mifepristone and FSH has been shown to inhibit premature LH surges effectively (4). However, the mechanism by which mifepristone inhibits gonadotropin secretion and ovulation has remained enigmatic. We (2) and other investigators (5) have speculated that the ability of mifepristone to delay ovulation is based mainly on its antigonadotropic effects. In addition, direct ovarian effects have been proposed $(6,7)$. In the current work, we evaluated the possible direct ovarian effects of the late-follicular phase administration of mifepristone by using a granulosa cell marker, inhibin $B$.

We investigated seven healthy, regularly menstruating women who received a single dose of mifepristone $(10 \mathrm{mg})$ on cycle day 10 or 11 (study day 0 ). Serum samples were collected daily for 7 days and then on day 10. Ovarian ultrasonography was performed on study days 0 and 6 . Serum concentrations of LH, FSH, progesterone, $\mathrm{E}_{2}$, mifepristone, and leptin, as well as follicular size in these subjects, have been described elsewhere (2). In the present work, that study was expanded upon by the use of a specific inhibin-B ELISA, devoid of cross-reaction with free inhibin- $\alpha$ subunits (8).

Mifepristone suppressed the circulating concentrations of FSH and inhibin B (Fig. 1A), as measured on the day after its administration. Because inhibin $\mathrm{B}$ has been proposed to be the main inhibitor of FSH secretion in the late follicular phase (9), these data confirm the antigonadotropic effect of mifepristone. However, the ability of mifepristone to suppress inhibin B and FSH clearly was associated with follicular size - transient suppression of inhibin

Received January 26, 2005; revised and accepted April 28, 2005. Supported by the Helsinki University Central Hospital Research Funds, the Finnish Cultural Foundation, and the Finnish Medical Foundation. Reprint requests: Oskari Heikinheimo, M.D., Ph.D., Department of Obstetrics and Gynecology, University of Helsinki, PO Box 140, SF-00029 Helsinki, Finland (FAX: 358-9-47174801; E-mail: oskari.heikinheimo@ helsinki.fi).
$\mathrm{B}$, but unchanged levels of FSH were encountered in women $(\mathrm{n}=3)$ with small $(<11 \mathrm{~mm})$ leading follicles on the day of mifepristone administration (Fig. 1B), suggesting a direct effect of mifepristone on the ovary. In contrast, profound and long-lasting suppression of both inhibin B and FSH was evident in women $(n=4)$ with larger leading follicles $(>11 \mathrm{~mm})$ on the day of mifepristone administration (Fig. 1C). On the basis of these observations, we propose that during the follicular phase, mifepristone acts mainly on the ovary when the follicular size is $<11 \mathrm{~mm}$, whereas at larger follicle sizes, the antigonadotropic effect of the drug becomes more important.

Previous studies have shown that mifepristone attenuates $\mathrm{E}_{2}$ secretion in response to exogenous FSH (10). Similarly, on the day of hCG administration after $\mathrm{COH}$ involving mifepristone and $\mathrm{FSH}$, circulating levels of $\mathrm{E}_{2}$ have been found to be lower in comparison with those associated with traditional $\mathrm{COH}$ (4). Thus, these previous data are suggestive of the fact that mifepristone acts on the ovary, whereas our results provide direct evidence. We speculate that mifepristone decreases the sensitivity of granulosa cells to FSH.

The effect of mifepristone on the health of the oocyte has been studied previously in women receiving clomiphene and hCG for $\mathrm{COH}$; administration of mifepristone 1 hour before hCG had no effect on the number of oocytes, rate of fertilization, or subsequent in vitro development of the embryos obtained (11). After $\mathrm{COH}$ using mifepristone, $\mathrm{FSH}$, and hCG, the mean number of oocytes retrieved was smaller, but their maturational stage more advanced when compared with oocytes harvested after a traditional $\mathrm{COH}$ protocol (4), suggesting a possible modulatory effect of mifepristone on oocyte maturation. However, no harmful effects on oocyte quality after mifepristone have been reported.

In conclusion, mifepristone appears to delay the midcycle gonadotropin surge at both central and ovarian levels. Before mifepristone is introduced into $\mathrm{COH}$ programs, its effects on follicle development should be carefully evaluated, and a regimen of mifepristone should be defined that is sufficient to inhibit preovulatory LH surges with minimal direct effects on the developing follicles. 
Circulating levels (mean $\pm \mathrm{SE}$ ) of $\mathrm{FSH}$ and inhibin $\mathrm{B}$ in seven healthy women after ingestion of $10 \mathrm{mg}$ of mifepristone on cycle day 10 or 11 (A). Concentrations of both FSH and inhibin B declined significantly from pre-mifepristone values to day 1 . The results also are displayed according to the size of the leading follicle $(<11 \mathrm{~mm} ; \mathrm{n}=3[\mathrm{~B}]$ or $>11 \mathrm{~mm} ; \mathrm{n}=4[\mathrm{C}])$ on the day of mifepristone administration. ${ }^{*} P<.05$ compared with the pretreatment values.

A

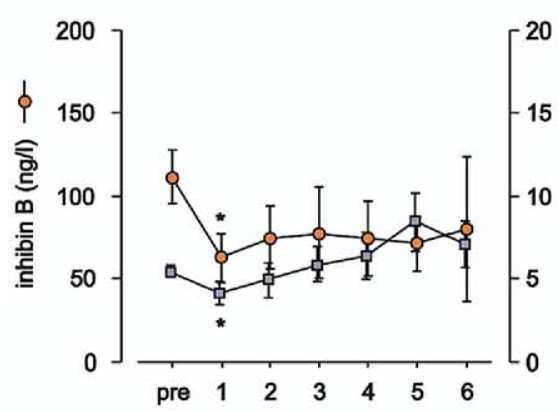

B

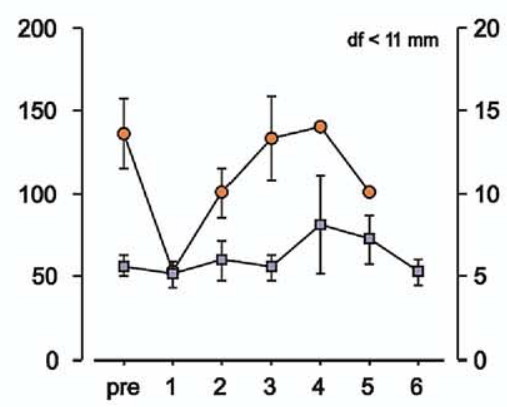

Days after mifepristone
$\mathrm{C}$

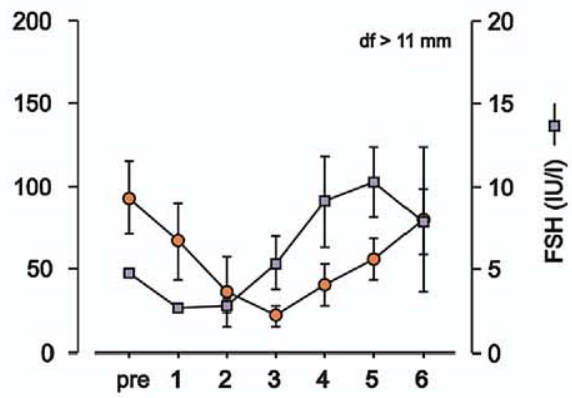

Heikinheimo. Mifepristone and inhibition of ovulation. Fertil Steril 2005.

Oskari Heikinheimo, M.D., Ph.D. ${ }^{\mathrm{a}, \mathrm{b}}$

Riikka Leminen, M.D. ${ }^{\mathrm{a}, \mathrm{b}}$

Taneli Raivio, M.D., Ph.D. ${ }^{\text {b,c }}$

${ }^{a}$ Department of Obstetrics and Gynecology,

${ }^{b}$ Department of Biomedicine, and ${ }^{c}$ Hospital for

Children and Adolescents, University of Helsinki,

Helsinki, Finland

\section{REFERENCES}

1. Marions L, Hultenby K, Lindell I, Sun X, Ståbi B, Gemzell Danielsson K. Emergency contraception with mifepristone and levonorgestrel: mechanism of action. Obstet Gynecol 2002;100:6570.

2. Leminen R, Raivio T, Ranta S, Oehler J, von Hertzen H, Jänne AO, et al. Late follicular phase administration of mifepristone suppresses circulating leptin and follicle-stimulating hormone-mechanism(s) of action in emergency contraception? Eur J Endocrinol 2005;152: $411-8$.

3. von Herzen H, Piaggio G, Ding J, Chen J, Song S, Bartfai G, et al. Low dose mifepristone and two regimens of levonorgestrel for emergency contraception: a WHO multicentre randomised trial. Lancet 2002;360:1803-10.

4. Escudero E, Boerrigter P, Coelingh Bennink H, Epifanio R, Horcajadas J, Olivennes F, et al. Mifepristone is an effective oral alternative for the prevention of premature LH surges and/or premature luteinization in women undergoing controlled ovarian hyperstimulation for in vitro fertilization. J Clin Endocrinol Metab 2005;90: 2081-8.

5. Baird D, Thong K, Hall C, Cameron S. Failure of oestrogen-induced luteinizing hormone surge in women treated with mifepristone (RU 486) every day for 30 days. Hum Reprod 1995;10:2270-6.

6. Croxatto H, Salvatierra A, Fuentealba B, Leiva L. Follicle simulating hormone-granulosa cell axis involvement in the antifolliculotrophic effect of low dose mifepristone (RU486). Hum Reprod 1995;10: 1987-91.

7. Kazem R, Messinis I, Fowler P, Groome N, Knight P, Templeton A. Effect of mifepristone (RU486) on the pituitary response to gonadotropin releasing hormone in women. Hum Reprod 1996;11:2585-90.

8. Groome N, Illingworth P, O'Brien M, Pai R, Rodger F, Mather J, et al. Measurement of dimeric inhibin $B$ throughout the human menstrual cycle. J Clin Endocrinol Metab 1996;81:1401-5.

9. Welt C, Pagan Y, Smith P, Rado K, Hall J. Control of folliclestimulating hormone by estradiol and the inhibins: critical role of estradiol at the hypothalamus during luteal-follicular transition. J Clin Endocrinol Metab 2003;88:1766-71.

10. Messinis I, Krishnan M, Kazem R, Khadilkar S, Templeton A. Effect of mifepristone on folliculogenesis in women treated with recombinant FSH. Clin Endocrinol 1997;46:309-14.

11. Messinis I, Templeton A. The effect of the antiprogestin mifepristone (RU 486) on maturation and in-vitro fertilization of human oocytes. Br J Obstet Gynaecol 1988;95:592-5. 